\title{
The Highly Peculiar Emission lines detected in Spectra of Extragalactic objects may be generated by Ultra-rapid Quasi-periodic Oscillations
}

\author{
Ermanno Borra ${ }^{\star}$
}

Department of Physics, Laval University, Quebec, Canada

Article Info
*Corresponding author:
Ermanno Borra
Laval University
Department of Physics
Physical and Optical Engineering
Quebec, Canada
Tel: 418-656-2131
E-mail: borra@phy.ulaval.ca

Received: January 24, 2019

Accepted: January 31, 2019

Published: February 6, 2019

Citation: Borra E. The Highly Peculiar Emission lines detected in Spectra of Extragalactic objects may be generated by Ultra-rapid Quasi-periodic Oscillations. Int J Cosmol Astron Astrophys. 2019; 1(2): 46-49. doi: 10.18689/ijcaa-1000112

Copyright: $\odot 2019$ The Author(s). This work is licensed under a Creative Commons Attribution 4.0 International License, which permits unrestricted use, distribution, and reproduction in any medium, provided the original work is properly cited.

Published by Madridge Publishers

\begin{abstract}
Extremely peculiar emission lines have been found in the spectra of some active galactic nuclei and quasars. Their origin is totally unknown. We investigate the hypothesis that they are generated from ultra-rapid quasi-periodic oscillations that may occur in jets or black holes, as predicted in a published theoretical paper. We conclude that, although not totally certain, this hypothesis is just as valid as the other highly peculiar hypotheses that have been previously made (e.g. blue shifts due to bulk motions close to the speed of light). We consider ways to further validate our hypothesis.
\end{abstract}

Keywords: Galaxies; Active Physical Data and Processes; Black hole physics; Physical Data and Processes; Line identification.

\section{Introduction}

The time domain is by far the least explored of all of the Astronomical physical domains. The difficulty with the short time domain is that it requires specialized and expensive instrumentation. It also requires extremely large quantities of data that are difficult to store and analyze. Furthermore, extremely fast time variations (e.g. 10-11 seconds) cannot even be detected with currently available instrumentation [1]. They proposed a very simple technique to find extremely rapid periodic time variations that have periods shorter than $10^{-11}$ seconds. Pulses separated by times shorter than $10^{-11}$ seconds generate periodic modulations in optical spectra that can be easily detected by carrying out Fourier transforms of the spectra [2]. They discuss the results of a Fourier transform analysis of the spectra of 0.9 million galaxies and 0.5 million stars and quasars in the Sloan Digital Sky Survey (SDSS). The Fourier transform analysis detected ultrarapid periodic modulations in the spectra of only 223 galaxies. There is no doubt that the signals are real, and not caused by instrumental effects, because plots of the periods as a function of red shift show that the periods increase with red shifts following two tight linear relations. These linear increases with red shift imply two base time periods of 1.02 $\times 10^{-13}$ seconds and $1.09 \times 10^{-13}$ seconds in the reference frames of the galaxies. Furthermore, none of the stars had signals at those time periods, while most of the stars had brighter apparent magnitudes than the galaxies and therefore gave spectra that had higher signal to noise ratios. Note that the fact that the galaxies are extended sources, while the stars are not, does not have any impact because the SDSS spectra used by [2] observe the cores of the galaxies with optical fibers that have a 3 arc seconds diameter.

[3] They extended the theoretical analysis of [1] to the case where the pulses are quasi-periodic. Quasi-periodic pulses are separated by the same average time when the average is carried out over long times; however the time separation between individual pulses varies at random. Computer simulations in [3] of the effect of quasi-periodic 
pulses show that they generate very narrow spectral lines if the average period is constant over the observation time. The lines are broadened if the average period is not constant over the observation time.

Extremely peculiar spectral emission lines have been found in the optical spectra [4] and X-ray spectra [5] of distant active galactic nuclei (AGNs) and quasars. The origin of these lines is totally unknown, but [5] hypothesize that they come from extremely blue shifted known spectral lines. They assume that the blue shift is due to extremely large bulk velocities (e.g. 0.75 the speed of light) or gravitational shifts from super massive black holes. Several surveys were carried out to find Lyman alpha emitters. For example [4] observed several luminous Lyman alpha emitters. They found other spectral lines that confirm the Lyman alpha assumption in all of them except two of them.

1. In this Work, we will consider the hypothesis that the discovered peculiar emission lines are generated from ultra-rapid quasi-periodic oscillations.

2. Summary of the theory of generation of emission lines from quasi-periodic oscillations.

This section contains a brief summary of the theory discussed in [3] that shows that ultra-rapid quasi-periodic pulses generate spectral features that look like emission lines.

[3] starts from the theory in [1] that models the time variations of an electric field $E(t)$, emitted by a source that sends $N$ periodic electric field pulses $V(t)$ that have a period $\tau$, by the convolution of $V(t)$ with a comb function $\sum_{m} \delta\left(t-t_{m}\right)$ made of the sum of delta functions $\delta\left(t-t_{m}\right)$ separated by $t_{m}$ $=(m-1) \tau$ where $m$ is an integer number. The electric field $E(t)$ is then given by the convolution of $V(t)$ and $\sum_{m} \delta\left(t-t_{m}\right)$

$$
E(t)=V(t) \otimes \sum_{m} \delta\left(t-t_{m}\right)
$$

The Fourier transform of the intensity, given by the square of the electric field in equation 1 , gives a spectrum as a function of frequency [1]. Then [1] shows that a quadratic detector, like a CCD, in a spectrograph detects a spectroscopic modulation given by

$$
S(\omega)=S_{1}(\omega)[\sin (\omega N \tau / 2) /(\sin (\omega \tau / 2))]^{2},
$$

Where $\omega=2 p v$ and $v$ is the frequency in the spectrum. This theoretical analysis is validated by the laboratory experiments of [6]. Then [1] models the electric field $E(t)$ of a very large number of periodic pulses separated by the constant period $\tau$ by the convolution of the comb function with a Gaussian that models the shapes of the individual pulses. The Fourier transform of this signal gives a frequency spectrum made of very narrow spectral lines, separated by a constant frequency separation $D V=1 / \tau$, having strengths that decrease with increasing frequency [3]. They starts from this analytical model to carry out numerical simulations of a Shah function where the separation between the pulses varies with random deviations from the period $\tau$. Here [3] shows the spectrum generated by this model and figure 3 in [3] shows the quasi-periodic electric field that generates this spectrum. The spectrum [3] is dominated by a very strong and narrow emission line, while the other lines are weakened below detection. The numerical modeling in [3] therefore demonstrates that very rapid quasiperiodic time variations generate a spectral feature that would be identified as an emission line in an astronomical spectrum. Because this emission line is generated at a location set by the average period $\tau$, it would probably be located at a peculiar spectral location and therefore be identified as a very peculiar spectral line.

\section{Generation from quasi-periodic oscillations of peculiar emission lines found in surveys}

Several objects have peculiar emission lines that have a totally unknown origin. For example [4] discuss the results of their spectroscopic follow-up of a large number of candidate luminous Lyman alpha emitters (LAEs) in the red shift range $5.7<z<6.6$. They discuss the spectra of 2 very peculiar LAEs (SR6 and VR7). They only find a strong emission line in their spectra, which they assume to be Lyman alpha at red shifts of $z$ $=5.676$ for SR6 and $z=6.532$ for VR7. However, while they searched for other spectral emission or absorption lines at the red shifts implied by the Lyman alpha assumption, they did not find any other line that validates the assumption. They clearly state that SR6 and VR7 are their most luminous objects, VR7 being the most luminous one, but do not have other spectral lines despite their luminosities; consequently the Lyman alpha assumption is only a hypothesis. They [7] also find a very peculiar LAE (MASOSA) that only has a very strong emission line but no other spectral lines and an undetected continuum. They compare them to Himiko $[8,9]$ which also has a similar spectrum with a single strong emission line but no other spectral features. Therefore there are several cases of astronomical objects that only have a single strong emission line but no other spectral line to confirm the Lyman alpha assumption.

They [5] found a peculiar narrow emission-line spectral feature in the X-Ray spectrum of the quasar PKS 0637-752 which is at the red shift $z=0.654$. This narrow emission-line is positioned at a spectroscopic energy location of $1.6 \mathrm{keV}$ in the quasar frame. Note that this $1.6 \mathrm{keV}$ energy is not the energy contained in the line. The energy in $\mathrm{keV}$ units is commonly used to identify the spectral location of the line in an X-Ray spectrum, while the wavelength is used in optical spectra. A spectral line had never been found at that X-Ray spectral location before. They [5] reject the hypothesis that it is caused by instrumental effects and explore other possible causes of this spectral line. Shortly after, [10] found an emission line in the radio-loud quasar PKS 2149-306 at an $X$-Ray spectral energy location of $17 \mathrm{keV}$ in the quasar frame which is at a red shift $z=2.345$. They conclude that the most likely explanation of the spectral line comes from the hypothesis that it is a Fe-K emission which is blue shifted by a bulk velocity of $0.75 c$, where $c$ is the speed of light. Since then, several detections of peculiar emission-lines in X-ray spectra have been found $[11,12]$. They are commonly assumed to be caused by known spectral lines (e.g. Fe-K emission line) that are extremely blue shifted or red shifted by extremely high gravitational or bulk velocity shifts. 
We will now investigate the hypothesis that these peculiar emission lines are caused by an extremely rapid quasi-periodic oscillation (QPO). The conclusion in [3] discusses the criteria needed to check the validity of a QPO spectral line. Although the discussion in [3] is centered on the spectra of Lyman $\alpha$ emitters, the same criteria apply to any spectral domain. One should simply look for other spectral features (e.g spectral lines or continuum breaks) at the same red shift or blue shift implied by the red shift or blue shift of the hypothetical known emission line (e.g. Fe-K emission). The presence of other spectral features would obviously invalidate the QPO hypothesis. The spectra of the objects in which a peculiar emission line has been found $[11,12,4,7,8,9]$ do not have other spectral features at the red shifts implied by the hypotheses that are commonly made (e.g. a blue-shifted Fe-K line). Consequently, this leaves the QPO hypothesis open.

\section{Discussion}

Quasi-periodic oscillations may occur in exotic objects like black holes or jets in AGNs and quasars. They [3] carry out an analysis that concludes that the extremely rapid periodic oscillations detected, by Fourier transforms of spectra, in the cores of galaxies are generated by jets in the core of the galaxies.

Quasi-periodic oscillations have already been found in a few black hole binaries that have masses of a few solar masses [13]. They have average periods as short as 3 milliseconds. The physics responsible for these quasi-periodic oscillations is unknown and many theoretical explanations have been proposed (see the introduction in [14] for a summary). The fact that rapid quasi-periodic oscillations have already been found in these exotic astronomical objects, although it obviously does not entirely validate it, strengthens the hypothesis that the emission lines found in several AGNS and quasars could be generated by extremely rapid oscillations. Super massive black hole binaries, that have masses of the order of $10^{8}$ solar masses, have been found [15-17] at the center of active galactic nuclei. It is therefore conceivable that these super massive binary black holes may generate the kind of ultra-rapid quasi-periodic pulses that we assume generate the emission lines. Because the physics responsible is unknown, it is conceivable that super massive black holes may generate quasi-periodic oscillations that have average periods far shorter than those found in binary black holes that have masses of the order of the mass of the sun [15].

The discussion in the previous paragraph is centered on black holes but, as mentioned at the beginning of this section, quasi-periodic oscillations may also occur in jets. Anything is possible in the relativistic jets that are in quasars and the cores of AGNs and the discovery of ultra-rapid (1.02 $\times 10^{-13}$ seconds and $1.09 \times 10^{-13}$ seconds) periodic modulations in the cores of 223 galaxies by [3] shows that relativistic jets can generate extremely rapid oscillations.

A QPO emission line is located, in a spectrum, at a frequency $v=1 / \tau$ (Equation 2), where $\tau$ is the average period of the pulsations, so that a QPO generated spectral line in the
X-ray spectral region at $10^{18} \mathrm{~Hz}$ would imply a value of the average period $\tau=10^{-18}$ seconds (see the discussion in section 2 and Equation 2). This value of the average period $\tau$ seems, at first sight, too short to make sense since it would require extremely high energy density values. However, the discovery of spectroscopic modulations coming from periodic pulses that have periods of $10^{-13}$ seconds in the cores of a small number of galaxies [3] shows that pulses having extremely short periods can be generated. The quasi-periodic oscillations coming from black hole binaries, having a few solar masses, that have already been found, also require extremely high energy density values. Furthermore, extremely short unresolved pulses $(<0.5 \mathrm{~ns}$ ) have been detected in the Crab pulsar [18]. They have a similar problem since this implies a brightness temperature higher than $2 \times 10^{41} \mathrm{~K}$.

One may, of course, wonder about what physical phenomena can possibly generate pulses separated by such extremely short times. The answer that we can give to this question is that anything is possible in jets or the extremely massive black holes that have been found at the center of many galaxies and that the discovery of quasi-periodic oscillations with time scales shorter than 2 milliseconds in binary black holes [13] strengthens the QPO hypothesis. Although there is no evidence that the average periods of the quasi-periodic oscillations having average periods shorter than 2 milliseconds, presently found in a small number of black holes that have masses of a few solar masses, decrease with increasing mass, it is conceivable that the time scales of the quasi-periodic oscillations decrease considerably in the super massive black holes, that have masses as large as thousands of billions of solar masses present at the center of galaxies. Emission lines may therefore be generated by ultrarapid quasi-periodic oscillations in the kind of super massive binary black holes that have been discovered in Galaxies. This discussion about the masses of black holes is irrelevant, if the quasi-periodic oscillations occur in jets, like the extremely rapid oscillations discussed [3].

\section{Conclusion}

At this stage, the hypothesis that the spectral lines in the spectra of AGNs and quasars are generated by ultra-rapid quasi-periodic oscillations has no greater validity than the other hypotheses discussed in $[10,12]$. However note that, while the fact that the spectra of the objects in which a peculiar emission line has been found do not have other spectral features at the red shifts implied by the hypothesis made by $[10,12]$ weakens their hypothesis, it strengthens the QPO hypothesis, The QPO hypothesis must be validated with further work. In principle, it should be validated with observations of the intensity as a function of time; however, time scales of the order of $10^{-18}$ seconds are too short to be detected with existing instrumentation. Presently, the only way to strengthen the QPO hypothesis is to obtain more spectra with very high signal to noise ratios. As discussed in section 3, one should simply look for other spectral features at the red shift of the emission line since their detection would 
invalidate the QPO hypothesis. However, while the nondetection of other spectral features would strengthen the hypothesis, it may not totally validate it, since these spectral features may be present but not be detected simply because they are too weak. The strength of these spectral features could be computer modelled to verify whether they are too weak to be detected is valid or not.

At first sight, the QPO hypothesis may seem very strange but, on the other hand, the other hypotheses presently considered (e.g. blue shifts at nearly the speed of light) are also highly peculiar. The fact that quasi-periodic oscillations having average periods as short as 3 milliseconds have already been found in black hole binaries [13] gives some credibility to the QPO hypothesis. Finally let us remember that super massive black hole binaries at the center of AGNs have also been found $[13,16,17]$ and that it is possible that super massive binary black holes may generate far more rapid oscillations. As mentioned several times in this paper, it is also possible that quasi-periodic oscillations may occur in jets and not black holes. The final conclusion is therefore that, although not certain, the QPO hypothesis is just as valid as the other hypotheses. More observations followed by computer modelling are needed to fully validate it.

\section{References}

1. Borra EF. Spectral signatures of ultra-rapidly varying objects in spectroscopic surveys. A\&A. 2010; 511: 4-8. doi: 10.1051/0004-6361/200913878

2. Borra EF. The discovery of Periodic modulations in the Optical spectra of galaxies, Possibly due to ultrarapid light bursts from their massive central black holes. ApJ. 2013; 774(2): 142-146. doi: 10.1088/0004-637X/774/2/142/ meta

3. Borra EF. Peculiar Emission Line Generation from Ultra-Rapid Quasi-Periodic Oscillations of Exotic Astronomical Objects. IASJ. 2017; 38(2): 23-32.

4. Matthee J, Sobral D, Darvish B, et al. Spectroscopic properties of luminous Lyman- $\alpha$ emitters at $\$ z \backslash$ lapprox $6-7 \$$ and comparison to the Lyman-break population. MNRAS. 2017; 472(1): 772-787. doi: 10.1093/mnras/stx2061

5. Yaqoob T, George IM, Turner TJ, Nandra K, Ptak A, Serlemitos PJ. A Peculiar Emission-Line Feature in the X-Ray Spectrum of the Quasar PKS 0637-752. ApJ. 1998; 505(2): 97-105. doi: 10.1086/311618
6. Chin SL, Francois V, Watson JM, Delisle C. Spectral modulation of two coherently separated femtosecond laser pulses. ApOpt. 1992; 31(18): 3383-3384. doi: 10.1364/AO.31.003383

7. Sobral D, Matthee J, Darvish B, et al. Evidence for Poplll-like Stellar Populations in the Most Luminous Lyman- $\alpha$ Emitters at the Epoch of Reionization: Spectroscopic Confirmation. ApJ. 2015; 808(2): 139-153. doi: 10.1088/0004-637X/808/2/139

8. Ouchi, $M$, Shimasaku $K$, Akiyama $M$, et al. The Subaru/XMM-Newton Deep Survey (SXDS). IV. Evolution of Lya Emitters from z $=3.1$ to 5.7 in the $1 \mathrm{deg}^{2}$ Field: Luminosity Functions and AGN. ApJS. 2009; 176(2): 301-330. doi: 10.1086/527673

9. Ouchi $M$, Ellis $R$, Ono $Y$, et al. An Intensely Star-forming Galaxy at $z \sim 7$ with Low Dust and Metal Content Revealed by Deep ALMA and HST Observations. ApJ. 2013; 778(2): 102-113. doi: 10.1088/0004-637X/778/2/102

10. Yaqoob T, George IM, Nandra K, Turner TJ, Zobair S, Serlemitos PJ. A Highly Doppler Blue shifted Fe-K Emission Line in the High-Red shift QSO PKS 2149-306. ApJ. 1999; 525(1): 9-14. doi: 10.1086/312327

11. Turner TJ, Miller $L$, Reeves $J N$, et al. Significant $X$-Ray Line emission in the 5-6 Kev B and of NGC 4051. ApJ. 2010; 712(1): 209-217. doi: 10.1088/0004$637 X / 712 / 1 / 209$

12. Bottacini $E$, Orlando $E$, Greiner J, et al. An extreme gravitationally Red shifted Iron line at $4.8 \mathrm{KeV}$ in Mrk 876. ApJ. 2014; 798(1): 14-27. doi: 10.1088/2041-8205/798/1/L14

13. Remillard RA, McClintock JE. X-Ray Properties of Black-Hole Binaries. ARA\&A. 2006; 44: 49-92. doi: 10.1146/annurev.astro.44.051905.092532

14. Varniere $P$, Vincent $F H$. Impact of inclination on quasi-periodic oscillations from spiral structures. A\&A. 2016; 591: 11-15. doi: 10.1051/0004$6361 / 201527711$

15. Rodriguez C, Taylor GB, Zavala RT, Peck AB, Pollack LK, Romani, RW. A Compact Supermassive Binary Black Hole System. ApJ. 2006; 646(1): 4957. doi: $10.1086 / 504825$

16. Boroson TA, Lauer TR. A candidate sub-parsec supermassive binary black hole system. Nature. 2009; 458(7234): 53-55. doi: 10.1038/nature07779

17. Deane RP, Paragi $Z$, Jarvis MJ, et al. A close-pair binary in a distant triple supermassive black-hole system. Nature. 2014; 511: 57-77. doi: 10.1038/ nature13454

18. Hankins TH, Eilek JA. Radio Emission Signatures in the Crab Pulsar. ApJ. 2007; 670(1): 693-701. doi: 10.1086/522362 